\title{
UM ESTUDO SOBRE O DESENVOLVIMENTO DE COMPETÊNCIAS EM AMBIENTE SIMULADO
}

Claudelino Martins Dias Junior (UFSC, e-mail: dias.jr@deps.ufsc.br) Bruno César Melo Moreira (IFMG, e-mail: bruno.melo@ifmg.edu.br) Eduardo Zarur Stosick (UFSC, e-mail: eduardo.stosick@gmail.com)

\section{RESUMO}

O presente trabalho descreve a percepção de alunos de graduação em administração no desenvolvimento de competências em ambiente simulado. Para tanto, construiu-se um estudo de caso, resultante das conclusões obtidas com a aplicação de questionário formado por questões abertas, às quais foram objeto de análise semântica, e questões fechadas, tratadas de forma a descrever os benefícios resultantes da participação em atividade de simulação. Os resultados preliminares apontam que os principais elementos formadores da competência gerencial centram-se na capacidade de integração e atualização de conhecimentos e no desenvolvimento de habilidades de liderança e de capacidade de organização, da mesma forma, comportamentos proativos e determinados constituem condições desejáveis sob a visão dos respondentes.

PALAVRAS-CHAVE: simulação, competência, aprendizagem.

\section{DEVELOPMENT MANAGEMENT COMPETENCES IN SIMULATION ENVIROMENTAL}

\section{ABSTRACT}

This paper describes the perception of students of the administration under graduate course, concerning the development of skills in a simulated environment. Therefore, a case study was built from the application of a questionnaire with open questions object of a semantic analysis and some others describe the benefits of the participation in the simulation activity. Preliminary results show that the main elements forming managerial competence are based on the ability to integrate and update knowledge and develop skills in leadership and also develop capacity of self organization. Besides that, proactive and determined behavior is a desirable condition in the perception of the students.

KEYWORDS: simulation, competence, learning.

\section{Introdução}

Nos últimos anos tem-se acompanhado o crescente desenvolvimento de tecnologias que permitem interconectar o indivíduo a um mundo novo e repleto de possibilidades. Tais avanços recentes nas áreas da computação, telecomunicações e tecnologias da informação estão mudando a maneira de se ver o mundo e cada vez mais têm alcançado as instituições de ensino (SAFANELLI; MOREIRA, 2011). 
Estas começam a perceber que as tradicionais formas presenciais de ensino, pautadas sobretudo nas aulas expositivas, não são suficientes para a grande demanda de formação que surge a todo instante, principalmente, em decorrência da evolução tecnológica e do surgimento de novos processos de trabalhos. Tal fato tem influenciado e motivado uma reformulação nos processos de ensino-aprendizagem buscando as necessidades dos alunos e do próprio mercado.

Esta mudança de perspectiva se torna ainda mais relevante em cursos predominantemente teóricos, como os cursos da área das Ciências Sociais aplicadas. O distanciamento da academia com a realidade provoca um verdadeiro abismo entre o que é aprendido em sala e os conhecimentos e habilidades que, de fato, os alunos necessitam na prática empresarial.

Com base nestas constatações, emergiu dentro dos programas de graduação e pós-graduação voltadas à área de gestão e negócios, o interesse na utilização de ferramentas que simulassem o ambiente empresarial, os simuladores organizacionais, que, simulando eventos que poderiam ser enfrentados no mundo real, permite proporcionar aos alunos aplicar os conhecimentos teóricos adquiridos nas situações vivenciadas (PAULA; BERGAMASCHI FILHO; SANTOS, 2012).

Medeiros e Schimiguel (2012) ressaltam que além dos benefícios que os elementos lúdicos promovem ao processo de aprendizagem, de tomada de decisões, no desenvolvimento cognitivo e na estimulação da criatividade, a utilização de jogos no processo de ensino-aprendizagem pode despertar um maior interesse por parte dos alunos, retirando-os da passividade pertinente aos modelos tradicionais de ensino, criando um processo ativo de construção do conhecimento e colaborando para uma diminuição na evasão escolar.

Sob este contexto o presente trabalho busca avaliar a percepção de alunos de um curso de graduação em administração, a respeito da intensidade dos benefícios alcançados no que concerne ao aprimoramento de conhecimentos, habilidades e comportamentos pela utilização de um simulador organizacional como estratégia de aprendizagem.

\section{2 - Desenvolvimentos de competências em ambientes simulados}

As simulações organizacionais surgiram em meados da década de 1950 (SAUAIA, 1990) derivados dos jogos existentes na área militar (MENDES, 2000). Sua implementação no meio acadêmico teve como premissa a possibilidade de se transferir os conceitos teóricos adquiridos pelos estudantes ao longo dos cursos para o campo prático, através de uma aprendizagem vivencial (BERNARD, 2006). Nesta aprendizagem vivencial, o papel principal e o centro do processo é o participante (aluno) que atua de forma ativa na construção do conhecimento (SAUAIA, 2010).

Devido às suas particularidades e dado a amplitude de seus propósitos de utilização, a adoção dos jogos ou simulações organizacionais tem possibilitado alcançar os mais diversos tipos de metas de aprendizagem e o desenvolvimento de competências caracterizadas em três grandes dimensões: aquisição de conhecimentos; desenvolvimento de habilidades e desenvolvimento de atitudes/comportamentos (SCHUMANN et al, 2001 apud VERSIANI; FACHIN, 2007).

De uma forma geral, observa-se que a definição de competência estaria relacionada à formação dos seguintes elementos: conhecimentos - consistiria dizer-se que o indivíduo domina o conteúdo, ou mesmo as premissas de base do conhecimento que pretenda aplicar ou fazer uso, ou seja, o quê "fazer"; as habilidades - estariam 
relacionadas à capacidade de prontidão observada em aplicar os conhecimentos, neste caso, conhecimentos tácitos, ou seja, seria o "saber fazer"; e os comportamentos ou atitudes necessárias à aplicação do conhecimento e da habilidade no indivíduo, em suma uma predisposição à ação “querer fazer”.

O Quadro 1 demonstra exemplos de conhecimentos, habilidades e atitudes/comportamentos requeridos em ambiente gerencial.

Quadro 1 - Competências gerenciais

\begin{tabular}{|c|c|c|}
\hline Conhecimentos & Habilidades & Atitudes/Comportamentos \\
\hline $\begin{array}{c}\text { Conceitos de estratégia } \\
\text { empresarial }\end{array}$ & Relacionamento interpessoal & $\begin{array}{c}\text { Postura contributiva } x \\
\text { competitiva }\end{array}$ \\
\hline $\begin{array}{l}\text { Conceitos de } \\
\text { mercadologia }\end{array}$ & Capacidade de processar informações & $\begin{array}{c}\text { Postura individual } x \text { Postura } \\
\text { coletiva }\end{array}$ \\
\hline $\begin{array}{l}\text { Conceitos de } \\
\text { contabilidade }\end{array}$ & Capacidade de trabalhar em equipe & $\begin{array}{l}\text { Enfoque nos objetivos e } \\
\text { resultados }\end{array}$ \\
\hline Conceitos de finanças & Raciocínio lógico & Postura de questionamento \\
\hline $\begin{array}{l}\text { Conceitos de recursos } \\
\text { humanos }\end{array}$ & Raciocínio matemático & $\begin{array}{l}\text { Flexibilidade e predisposição } \\
\text { à mudança }\end{array}$ \\
\hline $\begin{array}{l}\text { Conceitos de logística e } \\
\text { produção }\end{array}$ & $\begin{array}{l}\text { Capacidade de negociação Enfoque } \\
\text { na simplicidade }\end{array}$ & Enfoque na complexidade \\
\hline SABER & SABER FAZER & QUERER FAZER \\
\hline
\end{tabular}

Fonte: Elaborado a partir de Sauaia (2000) apud Versiani e Fachin (2007) e Junior et al (2013).

Sob estas premissas Oliveira e Sauaia (2008) argumentam que aprendizagem vivencial possibilita o desenvolvimento de competências a partir dos subsídios da teoria da aprendizagem vivencial de Kolb (1984) que concebe a aprendizagem como um processo e não decorrente dos resultados obtidos na forma de acúmulo de conhecimentos ou respostas a estímulos, conforme apresentado na Figura 1. Neste sentido, a construção de novos conhecimentos, habilidades ou atitudes/comportamentos exige que o participante (aluno) desenvolva quatro diferentes capacidades: experiência concreta (EC), observação reflexiva (OR), concepção abstrata (CA) e experimentação ativa (EA). (OLIVEIRA; SAUAIA, 2008, p.3).

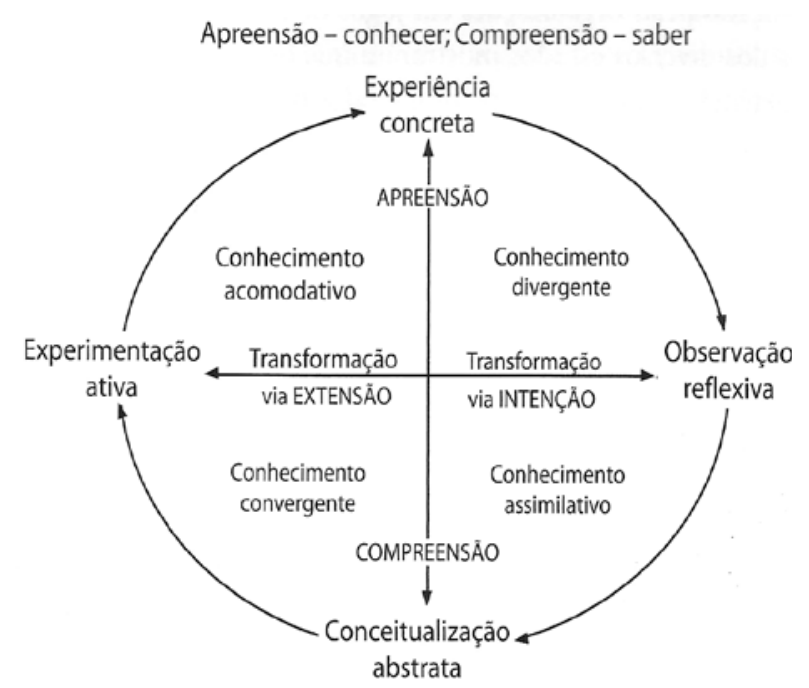

Figura 1 - Ciclo de aprendizagem vivencial.

Fonte: Sauaia (2010, p.xvii) 
Neste sentido, pautado na proposta de aprender fazendo, a aprendizagem vivencial tem início com um problema não estruturado que condiciona o participante a uma tomada de decisão inicial, configurando o ponto de partida da aprendizagem (EC). Da análise desta decisão primária (OR), e do confrontamento com teorias pré-existentes, conhecimento prévio e premissas inicias o participante (aluno) se torna capaz de criar conceitos que integrem suas observações de forma lógica em teorias (CA), conduzindo, então, a formação de novos conceitos ou do conhecimento novo, que posteriormente deverá ser testado (EA) por meio da adoção de novas decisões (OLIVEIRA; SAUAIA, 2008; SAUAIA, 2010). Desta forma, "o ciclo se completa e cada etapa encadeada se repete de forma a construir uma experiência vívida em cada participante, criando conhecimentos dinâmicos e sistêmicos numa contínua aprendizagem vivencial: fazer e refletir”. (SAUAIA, 2010,p xviii.).

Desta forma, os ambientes simulados, tal qual ressaltam Oliveira e Sauaia (2008), permitem ao aluno aprender, não apenas cognitivamente (conhecimento), mas também em termos do desenvolvimento da capacidade de implementar este conhecimento (habilidades) e em decorrência de decidir e assumir responsabilidade perante suas decisões (atitude/comportamento).

Adiante, apresenta-se a proposta metodológica de referência utilizada na construção do estudo.

\section{3 - Simulador Organizacional: um método de aprendizagem vivencial}

A experiência de utilização do Simulador Organizacional em análise funcionou dentro de um contexto acadêmico de ensino de graduação (Curso de Administração), onde o mesmo incorpora-se ao ementário de disciplina de cunho prático, denominada Laboratório de Gestão II. Neste sentido, suporta a proposta como um artefato metodológico, possibilitando o exercício do processo da tomada de decisão sobre investimentos necessários à potencialização de resultado econômico da atividade empresarial <http://www.simulab.com.br/ead/> .

Sendo função própria da atividade do profissional em Administração, o exercício de uma função gerencial consiste basicamente em trabalhar com cursos de ação que possa minimizar riscos envolvidos e maximizar resultados em uma situação real, a qual, num futuro não muito distante, estes profissionais serão colocados à prova.

Para efeito do Simulador Organizacional, tais riscos estão associados, principalmente, a: capacidade de previsão de demanda de produtos manufaturas e suas perspectivas de comercialização, com consequente dimensionamento de sua capacidade de produção; interpretação da ação dos concorrentes; adaptação de estratégia a ambiente macroeconômico em mutação e ajustamento de oferta a um preço que possa cobrir despesas incorridas dentro de exercícios financeiros.

Desta forma, de acordo com Sauaia (2010) o processo de ensino centrado na representação do conhecimento pelo professor passa a deslocar-se para a aprendizagem vivencial centrada na figura do participante.

Adiante, descrevem-se os procedimentos de análise que subsidiam a experiência de simulação dos grupos gestores.

\section{1 - Desenvolvimento do jogo}

A competição entre distintos grupos gestores se desenvolve, basicamente, sobre a premissa de alcance de melhores resultados econômicos/financeiros no interstício de 2 
(dois) anos ou ainda 8 (oito) trimestres de atividades de gestão simuladas. Esses mesmos grupos gestores partem de situações econômicas, financeiras, de produção e de mercado idênticas e demonstradas por resumidos instrumentos de gestão como: DRE (Demonstração de Resultado do Exercício); Balanço Patrimonial e Demonstrativo Operacional de Produção.

Neste sentido, a interpretação desses instrumentos condiciona a busca de objetivos qualitativos, que naturalmente passam a ser distintos, e dos quais devam ser transformados em metas orçamentárias (decisões de investimentos) que maximizem o retorno econômico sobre o capital investido por acionistas no respectivo período de gestão (8 trimestres), que, por conseguinte, representam "rodadas" de verificação de desempenhos grupais. A Figura 2 evidencia as decisões a serem tomadas trimestralmente por todos os grupos de trabalho no referido exercício de simulação.

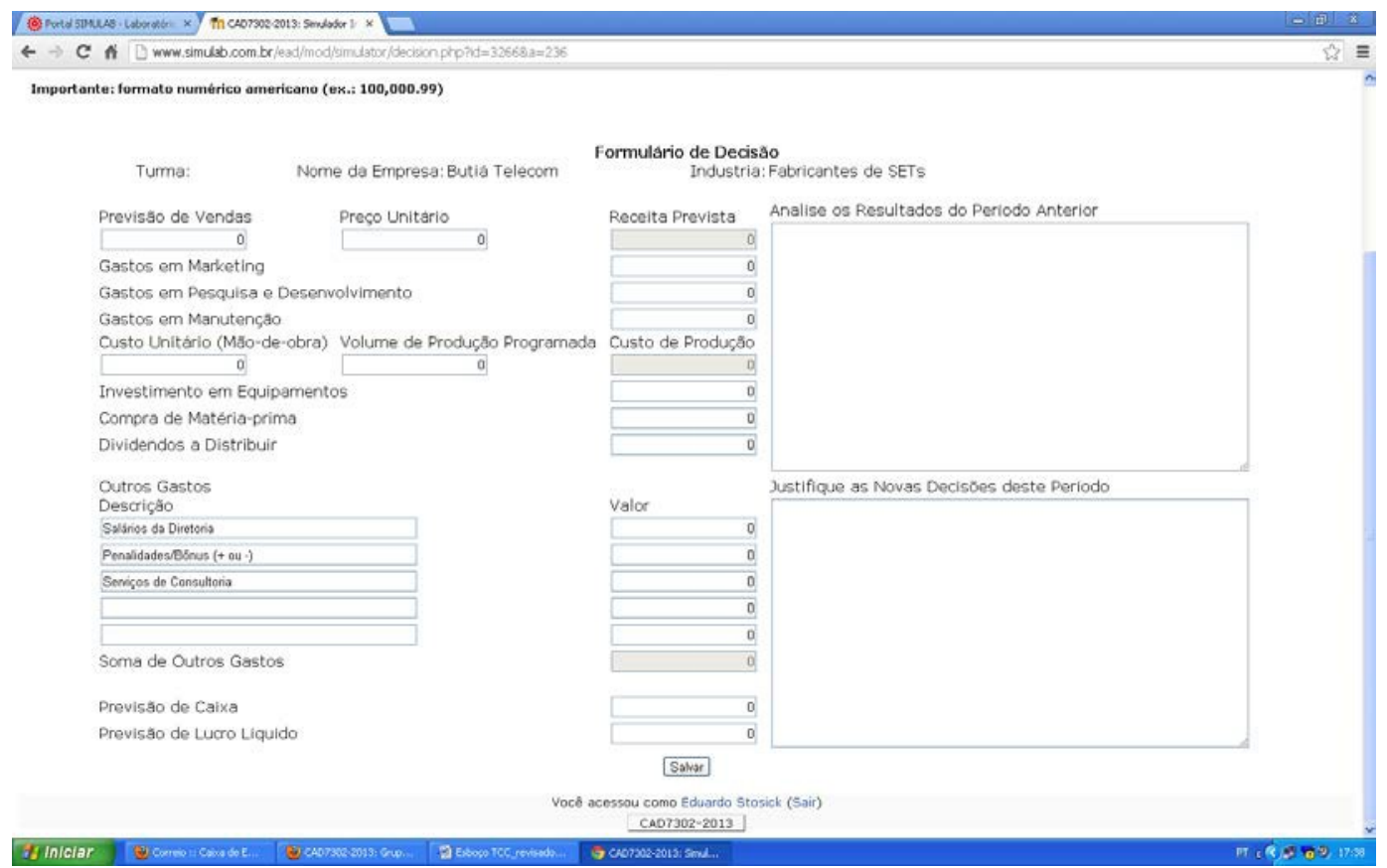

Figura 2 - Formulário de decisões trimestrais Simulab.

Fonte: < http://www.simulab.com.br/ead/mod/simulator/decision.php?id=3266\&a=236>

Ademais, os cálculos de eficiência (mercadológica, financeira e de produção) comuns ao cotidiano exercício da profissão de Administrador, para efeito do Simulador Organizacional, são mensurados a cada fechamento de exercício financeiro (1 ano ou 4 trimestres) e tidos como indicadores para reformulação da estratégia inicialmente adotada por cada grupo de trabalho (SAUAIA, 2010).

A seguir, os procedimentos metodológicos adotados são descritos na experiência de simulação em relato.

\section{4 - Procedimentos metodológicos}

Para avaliar a percepção dos alunos a respeito da intensidade dos benefícios alcançados pela utilização de um Simulador Organizacional utilizou-se da abordagem de estudo de caso em turma do Curso de Graduação Presencial em Administração da 
Universidade Federal de Santa Catarina composta por 32 (trinta e dois) alunos participantes do exercício de simulação, utilizado como artefato metodológico, ao longo de 1 (um) semestre letivo.

Separados em grupos que configuravam empresas fictícias os mesmos se viam diante da necessidade de tomar decisões gerencias que impactariam no futuro das empresas por eles gerenciadas e nas demais (concorrentes). Após um semestre de simulação os participantes foram solicitados a responder um questionário a respeito das experiências vivenciadas.

O questionário aplicado visou, portanto, avaliar se a prática simulada de um contexto organizacional poderia proporcionar ganho de conhecimento aos alunos. Com intuito de se caracterizar a amostra dos participantes, preliminarmente, foram coletadas as informações de cunho socioeconômico como: idade, sexo e formação acadêmica anterior.

Em seguida, na segunda parte do questionário, baseada na Escala Likert, foram apresentadas perguntas referentes à percepção dos respondentes quanto ao tipo de aula que confere a cada participante da disciplina um maior ganho de aprendizagem (Questão 4 - O tipo de aula que propicia a você uma aprendizagem com maior participação e aproveitamento parece ser?), a importância das características gerais da simulação para este ganho de aprendizagem (Questão 5 - Qual a importância de cada item para o seu aproveitamento?), os benefícios reais adquiridos com a prática da simulação (Questão 6 - Classifique a intensidade dos benefícios alcançados), e por fim, a opinião dos participantes quanto à validade da simulação para efeito do aprendizado proposto (Questão 7 - Qual sua opinião sobre o Jogo de Empresas?).

A seção 5 apresenta os resultados obtidos a partir do questionário proposto.

\section{5 - Apresentação e análise dos resultados}

Do total de 32 (trinta e dois) alunos matriculados na disciplina Laboratório de Gestão II e que participaram da simulação ao longo do semestre, 20 (vinte) responderam ao questionário proposto.

Os resultados preliminares obtidos demonstraram que a idade média dos participantes foi de 25 (vinte e cinco) anos. A amostra contou com a participação praticamente paritária entre homens e mulheres, todos com formação acadêmica conclusa em outras áreas do conhecimento ou em andamento no curso de administração.

Quando questionados a respeito de qual o tipo de aula lhes propicia uma aprendizagem com maior participação e aproveitamento, a grande maioria apontou as experiências com jogos como o modelo de aula mais significativa. Conforme exposto em Questão 4, em que notas foram atribuídas de acordo com: 1 (Concordo pouco) a 6 (Concordo muito), é possível observar que dentre as opções oferecidas, o modelo de Aula Expositiva foi considerado o que menos contribui para o aprendizado na opinião dos participantes, recebendo uma nota média de 3,4 pontos. Em seguida, com pontuação média de 3,7 pontos, o modelo de Seminários apresentou-se como segundo modelo que melhor proporciona aprendizado aos alunos avaliados. No entanto, com uma nota média bem mais expressiva, 5,05 pontos, o modelo baseado em jogos e simulações de empresas mostrou-se o mais adequado na garantia de um bom aproveitamento e aprendizagem.

Os resultados apresentados demonstram o claro interesse e reconhecimento por práticas pedagógicas alternativas à tradicional aula expositiva, sendo que um modelo 
pautado na vivência de ambientes simulados como nos jogos de empresas apresenta-se destacadamente mais atrativo e profícuo aos olhos dos participantes.

Já com relação às características da simulação empresarial vivenciada que mais se mostraram relevantes ao processo de aprendizado, foi possível identificar que os aspectos relativos ao contexto de competição entre as empresas fictícias, o interesse a respeito do assunto pelos participantes, as expectativas geradas com a vivência do jogo e a própria configuração do ambiente empresarial na simulação, se mostraram como elementos que mais impactaram no aproveitamento da disciplina e, consequentemente, na aprendizagem.

As características que apresentaram as maiores notas médias de um intervalo que compreendia 1 (Pouco importante) a 6 (Muito importante), destaca-se o contexto de competição entre as empresas fictícias, cuja avaliação média dos participantes foi de 4,95, apresentando-se assim como um dos elementos de maior importância para proporcionar um ambiente de aprendizagem que se assemelhe ao contexto real, proporcionando um elevado grau de aproveitamento dos participantes.

Para avaliar a intensidade dos benefícios obtidos com a participação na disciplina de Laboratório de Gestão II, propôs-se a Questão 6 (fechada) que avaliava a intensidade dos ganhos de conhecimentos, de habilidades e de comportamentos adquiridos ao longo da simulação. A Figura 3 apresenta os resultados que foram categorizados como "mais importante" (níveis de intensidade entre 4 e 6 pontos) e "menos importante" (níveis de intensidade entre 1 e 3 pontos).

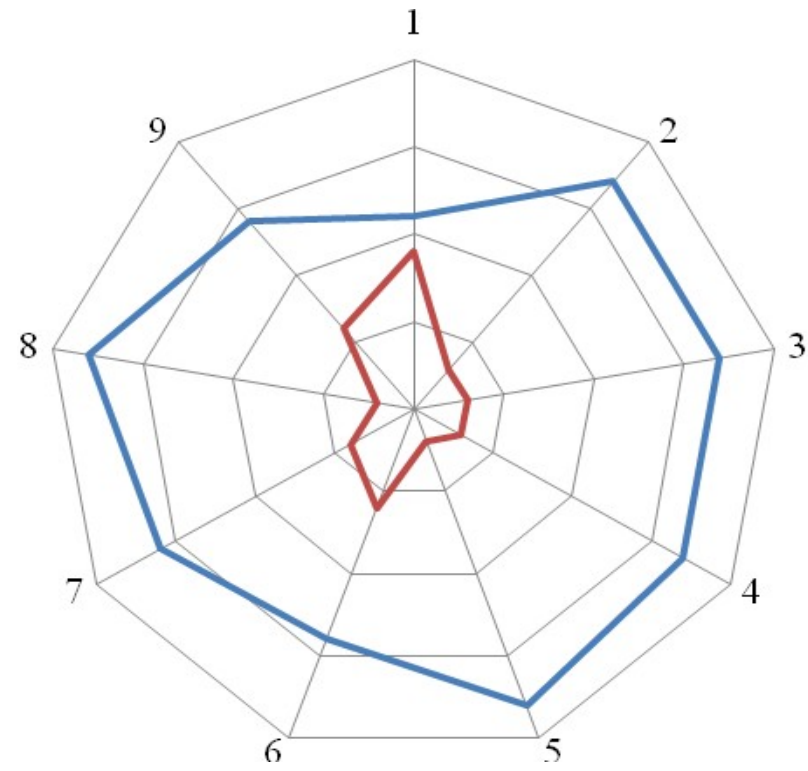

- mais importante

menos importante

Figura 3 - Níveis de importância dos elementos formadores de competências.

Fonte: elaboração própria.

O exercício de simulação mostrou-se relevante para praticamente todos os participantes da disciplina de Laboratório de Gestão II, com notas médias atribuídas acima de 4 (quatro) pontos, para todos os 3 (três) elementos formadores de competências (conhecimento, habilidade e comportamento). Assim, demonstrando-se proporcionalmente fortes os benefícios de aprendizado aos participantes.

Especificamente quando questionados a respeito da intensidade dos benefícios alcançados com relação aos conhecimentos, percebe-se que o objetivo do simulador 
organizacional fora alcançado. No entanto, depreende-se ainda, que pelo exposto em Figura 3, a aquisição de novos conhecimentos (eixo 1) não se fez tão relevante a uma proporcional parcela dos entrevistados (45\%). Não obstante, observa-se que a integração e a atualização dos conhecimentos (eixos 2 e 3) no contexto da disciplina de Laboratório de Gestão II são consideradas como “muito importantes” para uma parcela expressiva dos respondentes (85\%).

Por sua vez, o exercício de habilidades com a simulação, tais como a "prática de tomada de decisões" (eixo 5), a "prática de análise de problemas" (eixo 4) e a "prática do controle de resultados" (eixo 6), representaram respectivamente $90 \%$, 85\% e $70 \%$ de importância sob o ponto de vista dos respondentes. Desta forma, evidencia-se que a prática simulada permite proporcionar não apenas ganho de conhecimento aos alunos, mas na essência, permite criar/aperfeiçoar habilidades necessárias ao processo de tomada de decisão.

E em se tratando dos benefícios, oriundos do enfrentamento de situações problema vivenciadas com a simulação, sugere-se que a integração e a atualização dos conhecimentos, bem como o exercício de habilidades permitem aos alunos fortalecer sua capacidade de adaptar-se a novas situações (nota média 4,55), a capacidade de avaliar e explicar os resultados alcançados (nota média 4,65 ) e, dada à semelhança da simulação com o mundo real, fazer analogias entre o que se aprende na prática e o que ocorre de fato no contexto funcional das empresas (nota média 4,3).

Os questionamentos abertos colocados aos alunos entrevistados os induziam a descrever a importância do tipo de competência ou elemento formador desta competência que fora desenvolvido com o exercício de simulação. Dos resultados obtidos, sugere-se que as habilidades de liderança, de visão sistêmica, de organização, de planejamento e de trabalho em equipe foram respectivamente preponderantes. Ademais, a observância de comportamentos de proatividade e de determinação foram requisitos tidos como importantes pelos respondentes. A Figura 4 demonstra a participação de cada uma desses elementos apontados espontaneamente no formato de pergunta aberta, que do conjunto de respostas procedeu-se à análise semântica.

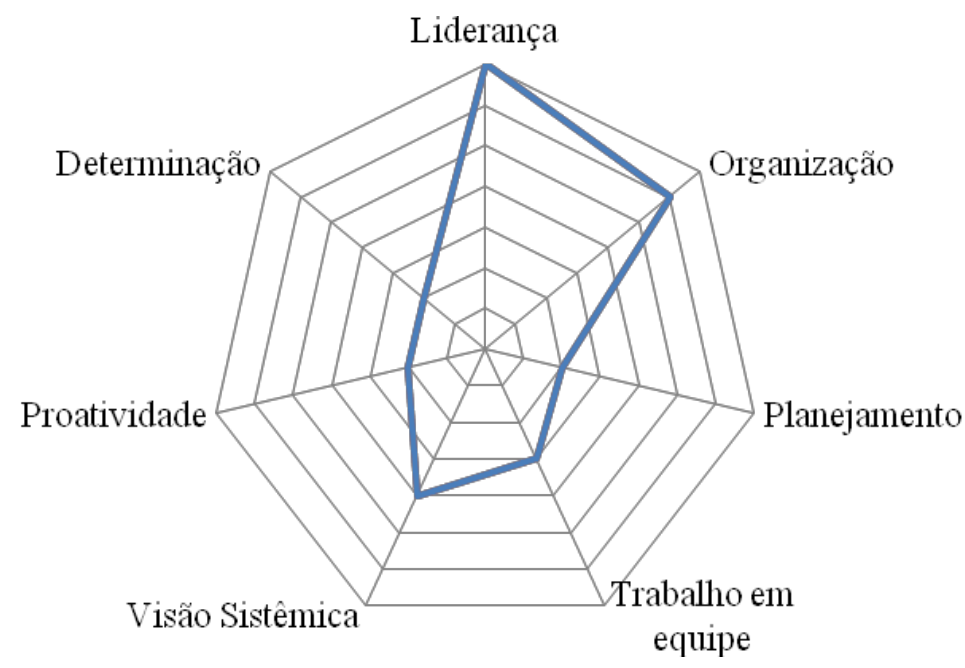

Figura 4 - Habilidades e comportamentos potencializados com a simulação. Fonte: elaboração própria. 
Por fim, os participantes foram questionados a respeito de sua percepção da simulação vivenciada. Dos respondentes, nenhum considerou o Simulador Organizacional do qual participaram como "fraco", o nível mais baixo da classificação. Neste sentido 20\% classificaram-no como "regular", 50\% classificaram-no como "bom" e 30\% como "muito bom", o nível mais alto da classificação. No computo final, a avaliação geral da simulação se mostrou favorável, reflexo da intensidade dos benefícios percebidos pela maior parte dos participantes.

\section{Conclusões}

O objetivo do presente trabalho foi capturar a percepção dos alunos graduandos da disciplina Laboratório de Gestão II em curso de administração, tencionando avaliar se benefícios gerados com a vivência gerencial, própria da atividade de simulação, estariam associados ao aprendizado e, consequentemente ao desenvolvimento de competências.

Após um semestre letivo gerenciando suas empresas fictícias e tomando decisões estratégicas de planejamento, marketing, produção, recursos humanos e finanças, os participantes foram indagados sobre quais os reais benefícios proporcionados por esta modalidade de ensino, em especial, sobre os elementos formadores das competências como: os conhecimentos (a partir de sua associação e efetiva utilização); as habilidades aprimoradas e os comportamentos diante de situações problemas. Observou-se que, para a grande maioria dos respondentes, os benefícios estariam relacionados mais intensamente a possibilidade de integrar os conhecimentos previamente adquiridos, o aprimoramento da habilidade de tomar decisões e a capacidade de avaliar e explicar os resultados alcançados.

Desta forma, foi possível demonstrar que a utilização da simulação organizacional auxilia no processo de formação de competências gerenciais, considerando que exista uma parcela de conhecimento novo gerado com essa prática de ensino e, principalmente, uma possibilidade real de agregação de conhecimentos já adquiridos em outras disciplinas durante o transcurso da graduação em administração.

Ademais, os outros elementos formadores de competências próprias do administrador como as habilidades de liderança, a capacidade de organização e a visão sistêmica, desenvolvidas e tidas como importantes na percepção dos respondentes, constituem importantes focos de atenção das organizações e, portanto, requisitos mais que desejáveis na formação do perfil dos administradores. Em paralelo, observa-se que a demonstração de atitudes proativas e determinadas diante de processos de gestão de natureza cada vez mais complexos, são atitudes (comportamentos) indispensáveis e de possível exercício em uma realidade presencial e virtual de ensino.

\section{REFERÊNCIAS BIBLIOGRÁFICAS}

BERNARD, R. R. S. Estrutura de Utilização dos Jogos de Empresas nos Cursos de Graduação em Administração e Ciências Contábeis do País e Avaliações Preliminares de uma Disciplina Baseada neste Método. In: Encontro da ANGRAD - ENANGRAD, 2006, São Luís - MA. Anais do XVII ENANGRAD, 2006.

JUNIOR, V. U.; PAULA, J. O.; PRUDENCIANO, R.; REZENDE, M. L. Identificação do Conhecimento, Habilidade e Atitude (CHA) dos coordenadores de curso de uma 
Instituição de Ensino Superior. In: SEGeT - Simpósio de Excelência em Gestão e Tecnologia, 2007, Rio de Janeiro: AEDB. p. 1-14.

KOLB, D. A. Experiential learning: experience as the source of learning and development. EUA: Prentice-Hall, 1984.

MEDEIROS, M. O.; SCHIMIGUEL, J. Uma Abordagem Para Avaliação De Jogos Educativos: Ênfase No Ensino Fundamental. Revista Novas Tecnologias na Educação, v. 10, p. 1-10, 2012.

MENDES, J. B. Utilização de jogos de empresas no ensino da contabilidade - uma experiência no curso de Ciências Contábeis da Universidade Federal de Uberlândia. In: Congresso Brasileiro de Contabilidade, 16, 2000, Goiânia. Goiânia: CFC, 2000. p. 119.

OLIVEIRA, M. A.; SAUAIA, A. C. A. Prontidão docente para aprendizagem vivencial: uma mudança de filosofia educacional por meio do Jogo de Empresas. In: XI SEMEAD - Seminários em Administração, 2008, São Paulo, SP. Anais do XI SEMEAD. São Paulo, SP: FEA/USP. p. 1-14.

PAULA, A. P. L.; BERGAMASCHI FILHO, E.; SANTOS, G. G. Análise da eficiência de ferramentas de simulação organizacional enquanto facilitadoras da aplicação de teorias para o curso de graduação em administração. In: Congresso Virtual Brasileiro Administração, 9, 2012, Florianópolis. Anais. Florianópolis: Convibra, 2012.

SAUAIA, A. C. A. Laboratório de gestão: simulador organizacional, jogo de empresas e pesquisa aplicada. São Paulo: Editora Malone, 2010.

SAFANELLI, A. S.; MOREIRA, B. C. M.. Empreendedorismo eletrônico: o uso da Educação Corporativa na formação de empreendedores. Revista Novas Tecnologias na Educação, v. 9, p. 1-11, 2011.

SCHUMANN, P. L. et al. A framework for evaluating simulations as educational tools. Developments in Business Simulation and Experiential Learning, v. 28, 2001. 